\title{
Effects of Incident UV light on the surface morphology of MBE grown GaAs
}

\author{
Daniel A. Beaton ${ }^{\mathrm{a}, *}$, C. Sanders ${ }^{\mathrm{a}}$, K. Alberi ${ }^{\mathrm{a}}$ \\ ${ }^{a}$ National Renewable Energy Laboratory \\ 16253 Denver West Parkway \\ Golden, CO 80401
}

\begin{abstract}
Light-assisted molecular beam epitaxy is a promising technique for improving the growth of metastable semiconductor alloys traditionally grown at low temperatures. The effect of photon irradiation on adatom incorporation dynamics is studied for GaAs homoepitaxy on vicinal surfaces. Irradiation is found to increase the temperature at which the growth mode transitions from layer-by-layer island nucleation to step flow growth and to alter the surface morphology. These surprising changes are discussed in the context of modification of adatom diffusion and incorporation processes.
\end{abstract}

Keywords: A1. Reflection high energy electron diffraction, A1. Roughening, A1. Surfaces, A3. Molecular beam epitaxy B2.

Semiconducting gallium arsenide

\section{Introduction}

The exploration of new semiconductor materials increasingly relies on growth techniques that operate far from equilibrium to overcome thermodynamic barriers to synthesis. In particular, low temperature molecular beam epitaxy (MBE) can extend the solubility limits of larger dopant atoms in a host crystal and promote incorporation at the expense of surface segregation[1]. The realization of $\mathrm{GaAs}_{1-\mathrm{x}} \mathrm{Bi}_{\mathrm{x}}$ and $\mathrm{Ga}_{1-\mathrm{x}} \mathrm{Mn}_{\mathrm{x}}$ As alloys, among other technologically important semiconductor alloys, has been achieved through this

\footnotetext{
*corresponding author, ph: 303384 7201, fax: 3033846430

Email address: daniel.beaton@nrel.gov (Daniel A. Beaton)
} 
approach $[2,3]$. However, low substrate temperatures can also reduce adatom mobility and produce films with higher lattice defect densities. The ability to grow these alloys with high crystalline quality is critical to incorporating them into devices. Additional methods are therefore needed to retain high crystalline quality while accessing non-equilibrium growth regimes.

The use of external stimuli to alter growth processes may provide an avenue for achieving high material quality at reduced substrate temperatures. Earlier work in this area focused on the use of energetic particles to alter the growth kinetics[4, 5, 6, 7]. However, that approach is difficult to implement uniformly and damages the film. Irradiation of the as-growing epitaxial film with above-bandgap photons is a potentially promising way to enhance dynamic growth processes without inducing structural degradation. Light-assisted MBE techniques have previously been shown to enhance p- and n-type doping concentrations and improve crystal quality in II-VI semiconductors[8]. Experimentally observed changes in the structural and electronic properties were suggested to arise from increased adatom mobility, selective species desorption, and/or the influence of photogenerated carriers on bonding $[9,10,11,12]$. Despite the initial technological success of this approach, the mechanisms underlying light-induced modifications to growth processes are as yet not well understood, in part because they have not been systematically studied with in-situ analytical techniques. A greater understanding of these mechanisms could lead to new approaches tailored to improve growth of metastable semiconductor alloys.

In this article we revisit the use of incident photons on as-growing epitaxial thin films as a function of growth temperature in order to understand its effects on adatom diffusion and surface morphology in a III-V semiconductor system. We apply in-situ reflective high energy electron diffraction (RHEED) measurements to probe the effects of photon irradiation on surface adatom diffusion. Results show that photon irradiation increases the temperature at which layer-by-layer growth transitions to step flow growth, which contradicts the expectation from previous reports that light stimulation improves adatom diffusion and mobility[10]. Ex-situ atomic force microscopy (AFM) measurements more clearly show that irradiation affects the surface morphology. We suggest two potential mechanisms to explain these observations. 


\section{Experimental procedures}

Samples were grown in an Omicron EVO25 MBE system on GaAs (001) substrates miscut $2^{\circ}$ towards $<111>$ A. A dual filament effusion cell was used for the gallium source and $\mathrm{As}_{2}$ flux was produced by a two-zone valved cracker source. Beam equivalent pressures (BEPs) were measured using an $i$ n-situ ion gauge, where gauge sensitivities were taken into consideration when calculating flux ratios[13]. The gallium flux and growth rate were further verified with RHEED intensity growth oscillations, as well as by epilayer thickness value obtained from the Pendellosung fringes in ex-situ X-ray diffraction (XRD) curves measured on specially prepared separate samples. The small variations in the fluxes of these sources from day to day will not affect the results presented below. Growth of all samples was carried out under group V rich conditions, with V:III flux ratios $>6$. The design of the growth chamber was modified to include a 4.5 " bakable sapphire viewport on a 10" bottom flange for direct optical access to the as-growing film surface. This viewport is frequently baked to remove any arsenic build up that would decrease optical transmission. Incident photon flux was provided using a broadband Xe lamp with significant output in the UV/blue wavelength range. The optical transmission of the sapphire viewport allows transmission of these short wavelengths. The Xe lamp is operated at $150 \mathrm{~W}$. It was partially collimated to form a spot size of approximately $25 \mathrm{~cm}^{2}$ on the sample. After losses, we estimate the incident power at the sample surface to be $5 \mathrm{~W} / \mathrm{cm}^{2}$.

The substrate was heated with a ceramic heater operated with a PID controller in conjunction with a thermocouple, which is situated between the substrate and the heater. Substrate temperatures were monitored using a $k$-Space BandiT band edge thermometry system with an accuracy of $\pm 1.0^{\circ} \mathrm{C}$, calibrated using the amorphous arsenic surface layer and native oxide desorption temperatures identified with RHEED. Note that this calibration procedure results in an overall absolute error of up to $10^{\circ} \mathrm{C}$ from the actual substrate temperature, it is does not contribute to any relative error in substrate temperatures for the measurements discussed below. The set point of the PID control is adjusted to achieve the desired substrate temperature as measured by the BandiT system. The small amount of sample heating $\left(5-10^{\circ} \mathrm{C}\right)$ due to irradiation by the Xe lamp was compensated for by an appropriate minor reduction in the temperature set point in order to achieve the desired substrate temperatures.

Substrates were outgassed at $300^{\circ} \mathrm{C}$ in an ultra-high vacuum (UHV) buffer 
chamber and the oxide was thermally desorbed in the growth chamber under $\mathrm{As}_{2}$ overpressure. For RHEED intensity oscillation measurements, a thick $(500 \mathrm{~nm}) \mathrm{GaAs}$ buffer layer was first grown without illumination at $580^{\circ} \mathrm{C}$. RHEED oscillations were measured with and without illumination as a function of substrate temperature. For surface morphology measurements by AFM, a $300 \mathrm{~nm}$ GaAs buffer was first grown without illumination at $\mathrm{T}_{\text {substrate }}=580^{\circ} \mathrm{C}$. This was followed by a brief growth interruption to allow the surface to smooth and ensure similar starting morphologies for all samples. The topmost GaAs epi-layers were grown to a thickness of $300 \mathrm{~nm}$ as a function of substrate temperature and photon flux. The substrate temperature was immediately quenched after growth to preserve the surface morphology of the epi-layer growth surface. Surface morphologies were probed with a Veeco Dimensions IIIa AFM system in tapping mode under ambient conditions using tips with a nominal radius of curvature of $10 \mathrm{~nm}$.

\section{Results and Discussion}

Oscillations in RHEED intensity arise from scattering of the electron beam via layer-by-layer island nucleation and coalescence on the terraces of the vicinal surface[14]. Disappearance of the oscillations at higher temperatures on mis-cut substrates indicates that the adatom diffusion length, $\lambda$, has exceeded the average terrace width, $l$. At this point growth proceeds in a step flow mode in which adatoms predominantly incorporate at step edges. Figure 1 shows RHEED oscillations measured under dark and illuminated conditions on $2^{\circ}$ mis-cut substrates at various temperatures. Disappearance of RHEED intensity growth oscillations under dark conditions is consistent with with observations reported in the literature for this substrate mis-cut[15]. Oscillations were consistently observed to disappear at a higher temperature of at least $10^{\circ} \mathrm{C}$ under illuminated as compared to under dark growth conditions. These values were verified by increasing and decreasing the substrate temperature repeatedly through the transition and by repeating the experiment on multiple samples. The measurements were also taken out of sequence to remove any systematic error. It is important that note that this transition does not occur abruptly under illuminated conditions and oscillations persist, albeit at a lower intensity over a range of temperatures above the transition temperature observed under dark growth conditions. The transition from predominantly layer-by-layer growth to step-flow has been shown to occur over a small temperature range[16]. Under illuminated 


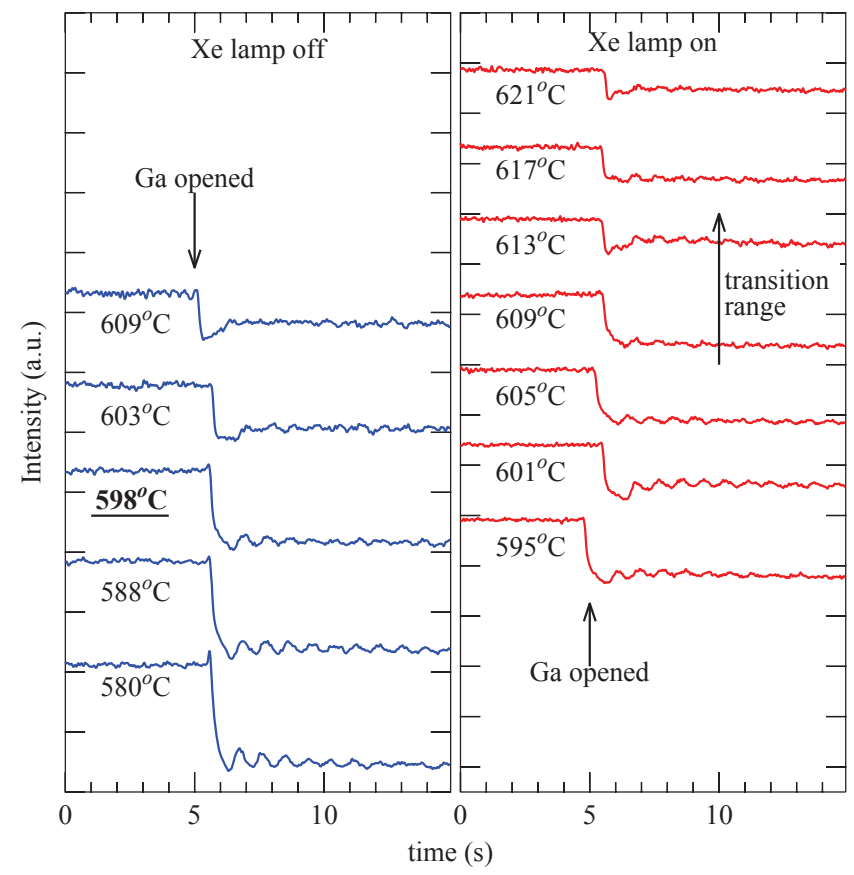

Figure 1: RHEED intensity growth oscillations for a substrate miscut $2^{\circ}$ to $\langle 111\rangle \mathrm{A}$ as a function of substrate temperature. The disappearance of the oscillations indicates that the surface adatom diffusion length has exceeded the average terrace width. The transition temperature is underlined and bolded for clarity for the dark growth conditions sample, and we estimate an uncertainty of $\pm 4^{\circ} \mathrm{C}$.

growth conditions this lack of abruptness in the transition is enhanced. The difference in the transition temperature between dark and illuminated conditions is greater than the difference due sample heating under illumination. Furthermore, additional unaccounted for thermal energy introduced to the growth surface from illumination would have the opposite effect of shifting the transition to lower temperatures. These results demonstrate that illumination does not increase adatom diffusion length contrary to expectation.

While the relatively weak persistence of RHEED intensity growth oscillations to higher substrate temperatures under illuminated conditions gives some indication that incident light affects the adatom surface kinetics, changes in the surface morphology provide more convincing evidence of this affect. Figure 2 shows the surface morphologies of a set of $300 \mathrm{~nm}$ thick GaAs films grown at various substrate temperatures with and without photon irradiation. The film surfaces are observed to progress with increasing temperature 
from well-defined mounds elongated along the [110] direction to wider moundlike features, and finally to an isotropically flat surface. Elongation of the mounds in the [011] direction is due to preferential Ga adatom diffusion in this direction. The degree of roughness and the nature of the surface morphology observed here are generally similar to those reported in Ballestad et al.[17]. The surface morphologies of the non-illuminated samples are similar to those of illuminated samples grown at higher $\left(+20-30^{\circ} \mathrm{C}\right)$ temperatures. This temperature difference is similar to the observation of a shift in the step flow growth transition temperature discussed above.

Power spectral densities (PSDs), calculated from AFM images of the samples in Fig. 2, provide additional information related to the growth modes. The PSD spectra, shown in Fig. 3, are from calculations along the [110] direction for $1 \times 1 \mu \mathrm{m}$ and $6 \times 6 \mu \mathrm{m}$ AFM scans. The general form of the PSD is predicted by continuum growth equation models, e.g. $\delta_{t} h \propto \nabla^{2} h+\nabla^{4} h+\cdots[18]$. A defining feature of PSD spectra for GaAs grown under step flow growth conditions is the tendency of the PSDs to display a slope of -2 , indicating a continuum growth equation dominated by the first order linear term[17]. The second order linear term, $\nabla^{4} h$, dominates in PSDs of surfaces with higher step edge densities, such as those resulting from predominantly layer-by-layer growth. Lines with slopes $x^{-4}$ and $x^{-2}$ are shown in Fig. 3 as a guide to the eye. The relative shapes of the PSD spectra from samples grown under dark conditions (Fig. 2a, c, and e) agree well with the PSD spectra from illuminated samples grown at $20-30^{\circ} \mathrm{C}$ higher temperatures (Fig. 2b, d, and f) indicating similarities in adatom surface kinetics. The PSD spectra from the sample pairs grown at low and intermediate temperatures more closely follow the $x^{-4}$ slope, which is expected for the higher step edge densities formed during layer-by-layer growth. The lower temperature pair exhibits higher relative weights at all spatial frequencies due to their relatively rougher surfaces. The spectral weights shift to shorter spatial frequencies at higher growth temperatures before transitioning closer to the $x^{-2}$ slope at the onset of predominantly step flow growth at the highest temperatures. The incomplete shift from a slope of -4 to -2 in the illuminated sample in the highest growth temperature pair is consistent with the persistence of weak RHEED intensity growth oscillations and island formation during growth above the transition to predominantly step flow growth.

Figure 4 shows the distribution of surface inclination angles obtained from the AFM images in Fig. 2. The inclination angle is defined, for each pixel in the AFM scan, as the angle between the normal to a plane fitted 

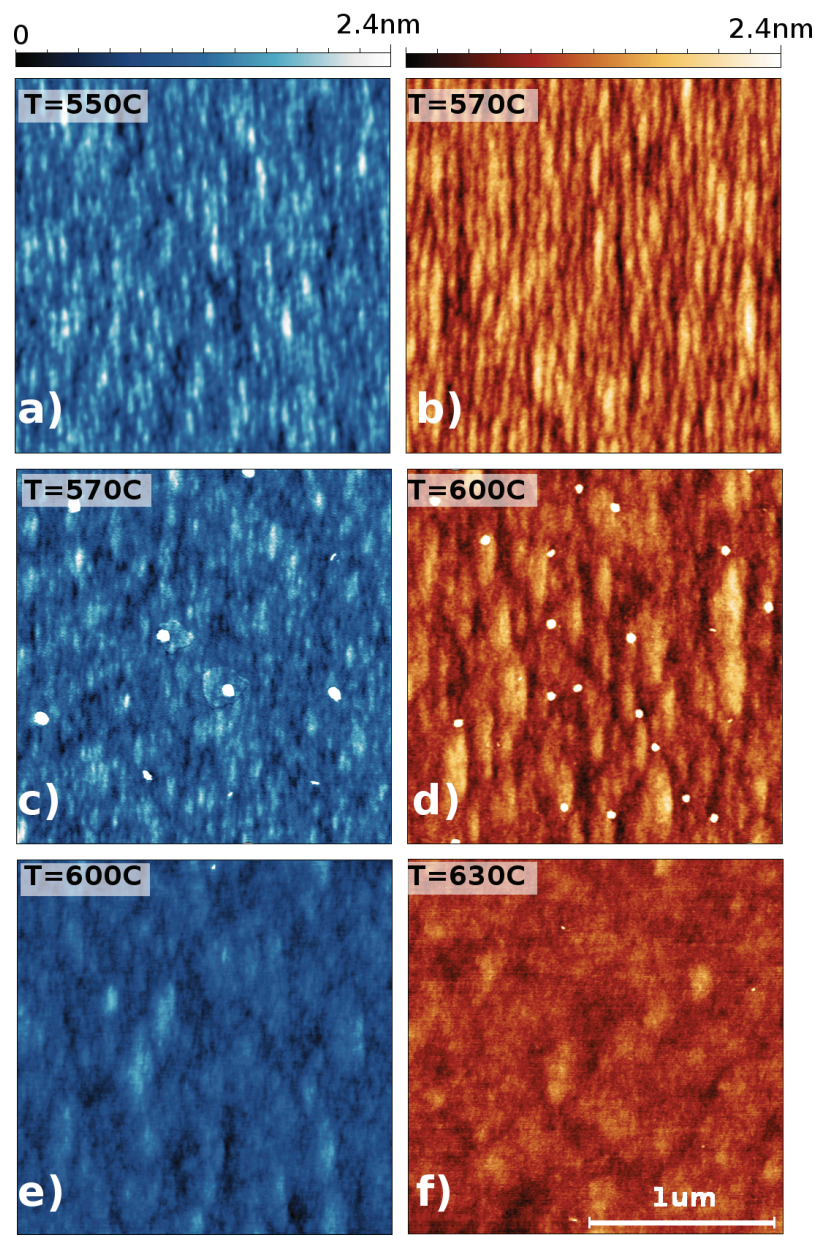

Figure 2: Set of AFM scans for a series of samples grown below (a,b), near $(\mathrm{c}, \mathrm{d})$ and above $(\mathrm{e}, \mathrm{f})$ the RHEED intensity growth oscillations transition temperature. Samples $\mathrm{a}, \mathrm{c}$, and e were grown without incident photon flux and samples $\mathrm{b}, \mathrm{d}$, and $\mathrm{f}$ were grown under illumination from a broadband Xe lamp. Comparable surface morphologies are found for increased substrate temperatures when samples grown under illumination. RMS roughnesses are: a) $0.32 \mathrm{~nm}$, b) $0.32 \mathrm{~nm}$, c) $0.30 \mathrm{~nm}$, d) $0.29 \mathrm{~nm}$, e) $0.21 \mathrm{~nm}$, and f) $0.17 \mathrm{~nm}$. 


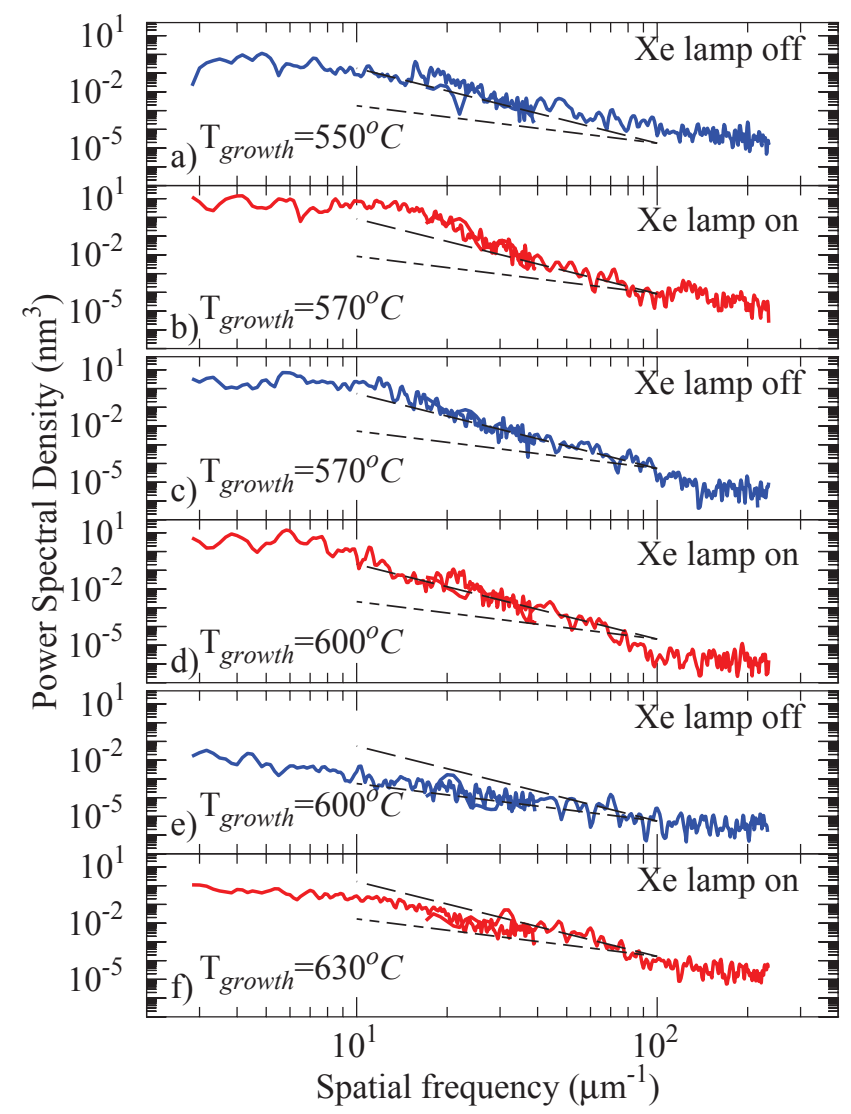

Figure 3: Power spectral densities of the sample set characterized by AFM in Fig. 2. All PSDs are shown on the same vertical scale and the dashed lines with slopes $x^{-4}$ (dash) and $x^{-2}$ (chain) are shown as a guide to the eye. Similarities can be seen between the sample pairs grown with and without incident radiation. 


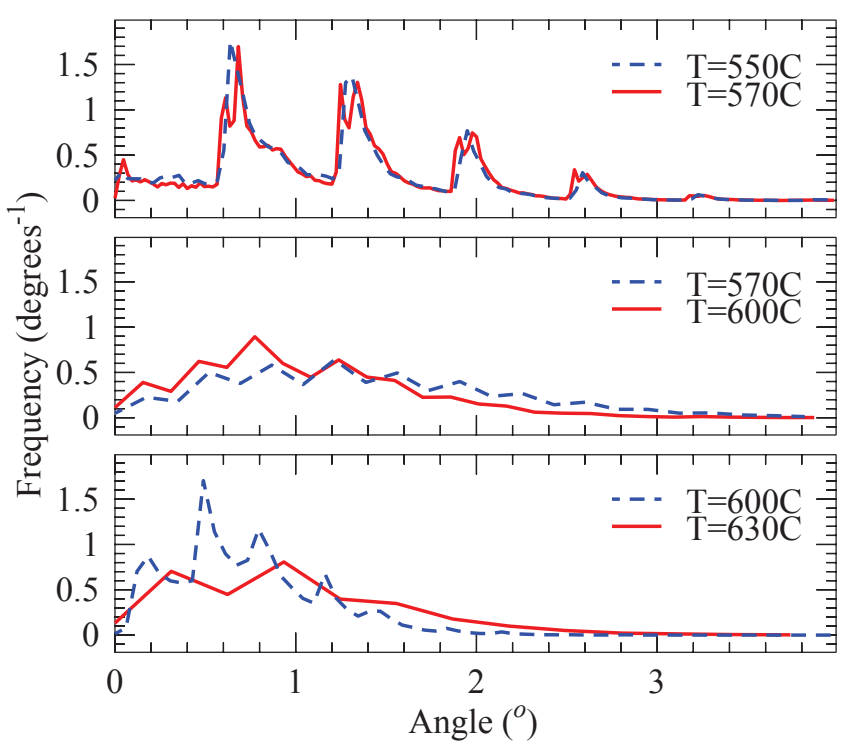

Figure 4: Distribution of surface angles present in the morphology of the sample set shown in Fig. 2 of non-illuminated (dashed blue curves) and illuminated (red solid curves) epi-layers.

to the surface at that point and the normal to the scan plane. Note that the scan plane is parallel to the $2^{\circ}$ miscut of the substrates. There are clear similarities between the sample pairs grown with and without illumination and clear distinctions between the sets grown at low, intermediate, and high temperatures. The lowest temperature pair, grown well below the threshold for step-flow growth, exhibits a strong preference for specific angles, including a non-trivial incidence of relatively steep angles corresponding to narrow atomic terraces typically present in mounds. At higher growth temperatures, close to the threshold for step-flow growth, the preference for specific angles is weakened and there is a shift in the angular distribution to smaller angles corresponding to wider atomic terraces. The highest temperature sample pair, grown above the threshold for step-flow growth, exhibits a distribution of angles skewed towards the lowest values, consistent with the flat morphology expected in step-flow growth. The distinct similarities exhibited in both the PSD spectra and surface angle distribution data in the samples pairs shown in Fig. 2a/b, c/d, and e/f strongly suggests that illumination shifts the adatom surface dynamics in a similar fashion as would a decrease in substrate temperature of $20-30^{\circ} \mathrm{C}$. 
An increase in the GaAs layer-by-layer to step flow growth mode transition temperature under broadband illumination could arise from a few potential effects. The first possible mechanism is a change in the adatom kinetics, e.g. gallium adatom surface diffusion lengths. Following the initial arguments by Neave $e t$ al., step flow growth dominates when the adatom diffusion length becomes equal to or greater than the average terrace width[19]. The surface adatom diffusion coefficient is given by:

$$
D=D_{o} e^{-E_{D} / k T}
$$

which is related to the diffusion length by the Einstein relation, $\lambda^{2}=2 D \tau$. Here, $E_{D}$ is the surface diffusion activation energy, and $\tau\left(\tau=J_{G} a / N_{s}\right.$, where $J_{G} a$ is the flux of gallium atoms and $N_{s}$ the density of surface sites) is the average arrival time of Ga adatoms at a specific surface site. Our observation that illumination leads to an increase in the transition temperature at which RHEED oscillations disappear suggests a small increase in $E_{D}$. In other words, the observation of a decrease in surface adatom diffusion indicates that the irradiation does not simply add thermal kinetic energy to the adatoms at these growth temperatures. Evidence of reduced adatom diffusion seemingly contradicts previous conclusions that light stimulation enhances adatom surface mobility during the epitaxy of II-VI semiconductors[10]. This effect was attributed in part to preferential desorption of chalcogenide adatoms during irradiation, leaving a more metal rich surface on which adatoms are free to diffuse over longer distances. Migration enhanced epitaxy (MEE) operates by a similar principle, and both MEE of III-V semiconductors and light stimulated MBE of II-VI semiconductors was found to produce smoother growth surfaces compared to conventional MBE[10, 20, 21]. This explanation also does not explain the increase in roughness that is observed in the present experiments.

The other possible mechanism that could be responsible for the increase in the step flow transition temperature under broadband illumination is blocking of adatom incorporation sites at step edges by photogenerated carriers. Step edges running parallel to the [110] direction (referred to as B step edges) are terminated by As atoms and provide $\mathrm{SP}^{3}$-like dangling bonds that act as preferential incorporation sites for Ga[21]. Previous exploration of hydrogenassisted MBE suggests that hydrogen may passivate these dangling bonds and thereby suppress the onset of step flow growth from B step edges by approximately $20^{\circ} \mathrm{C}[15,22]$. Growth in the present case was carried out on surfaces miscut toward the A direction, on which the step edges running 
in the [110] direction (referred to as A step edges) do not act as preferential incorporation sites. However, slight roughness in the step edges along the A direction will produce some B step edges that will support step flow growth[21]. In fact, only a relatively small degree of hydrogen surface coverage $(\simeq 1 \%)$ is needed to alter the growth mode in semiconductors[23]. A similar but perhaps distinct mechanism of dangling bond passivation by photogenerated carriers may also explain the modest $\left(\gtrsim 10^{\circ} \mathrm{C}\right)$ shift in the step flow transition temperature that is observed under light stimulation. Further investigation is required to delineate these potential mechanisms.

\section{Conclusion}

In summary, the influence of photon irradiation on adatom kinetics during GaAs homoepitaxy at intermediate to high growth temperatures has been systematically investigated by in-situ measurements of the layer-by-layer to step flow growth transition temperature and ex-situ surface morphology measurements by AFM. Photon irradiation was found to increase both the layerby-layer to step flow growth mode transition temperature and the morphological evolution of the surface features by $20-30^{\circ} \mathrm{C}$. These changes may be caused by either an increase in the energy barrier to adatom diffusion or by the blocking of incorporation sites by photogenerated carriers. Such a detailed level of understanding of surface irradiation and its effects on surface adatom kinetics could lead to growth approaches specifically designed to overcome barriers to the growth of metastable semiconductor alloys.

\section{Acknowledgements}

We acknowledgement the financial support of the Department of Energy Office of Science, Basic Energy Sciences under DE-AC36-OSGO-28308

[1] R.F.C. Farrow, J. Vac. Sci. \& Technol. B 1, 222 (1983)

[2] S. Tixier, M. Adamcyk, T. Tiedje, S. Francoeur, A. Mascarenhas, P. Wei, and F. Schiettekatte, Appl. Phys. Lett. 822245 (2003

[3] H. Ohno, A. Shen, F. Matsukura, A. Oiwa, A. Endo, S. Katsumoto, and Y. Iye, Appl. Phys. Lett. 69, 363 (1996)

[4] J.L. Zilko, J.E. Greene, J. Appl. Phys. 51, 1549 (1980) 
[5] J.E. Greene, S.A. Barnett, J. Vac. Sci. Technol. 21, 285 (1982)

[6] W. Ensinger, Nucl. Instr. and Meth. in Phys. Res. B 127, 796 (1997)

[7] D.M. Mattox, J. Vac. Sci. Technol. A 7, 1105 (1989)

[8] R.N. Bicknell, N.C. Giles, J.F. Schetzina, C. Hitzman, J. Vac. Sci. Technol. A 5, 3059 (1987)

[9] J.D. Benson, D. Rajavel, B.K. Wagner, R. Benz II, C.J. Summers, J. Crys. Grow. 94, 534 (1989)

[10] Y. Marfaing, Semicond. Sci. Technol., 6, A60 (1991)

[11] N. Matsumura, T. Fukada, J. Saraie, J. Crys. Grow. 101, 61 (1990)

[12] H.H. Farrell, R.E. Nahory, J.P. Harbison, J. Vac. Sci. Technol. B 6, 779 (1988)

[13] V.V. Preobrazhenskii, M.A. Putyato, O.P. Pchelyakov, and B.R. Semyagin. J. Crys. Grow., 201/202, 170 (1999)

[14] Ayahiko Ichimiya and Philip I Cohen. Refelction High Energy Electron Diffraction. Cambridge University Press, New York, 2004.

[15] H. Asahi, T. Hisaka, S.G. Kim, T. Kaneko, S.J. Yu, Y. Okuno, S. Gonda, Appl. Phys. Lett., 61:1054, 1992

[16] B. A. Joyce, J H Neave, J. Zhang, D. D. Vvedenskyt, S. Clarke, K. J. Hugill, T. Shitara, and A. K. Myers-Beaghton, Semicond. Sci. Technol. 5, 1147, (1990)

[17] A. Ballestad, B.J. Ruck, J.H. Schmid, M. Adamcyk, E. Nodwell, C. Nicoll, and T. Tiedje, Phys. Rev. B 65, 205302 (2002)

[18] A.L. Barabasi and H.E. Stanley. "Fractal concepts in surface growth" Cambridge University Press, Cambridge, United Kingdom, 1995.

[19] J. H. Neave, P. J. Dobson, B. A. Joyce, and J. Zhang, Appl. Phys. Lett. $1001(1985)$

[20] M. Kitagawa, Y. Tomomura, K. Nakanishi, A. Suzuki, and S. Nakajima, J. Crys. Grow. 101:52, 1990 
[21] Y. Horikoshi, M. Kawashima, and H. Yamaguchi, Jpn. J. Appl. Phys., 27:169, 1988

[22] Y. Okada, T. Fujita, and M. Kawabe, Appl. Phys. Lett., 67:676, 1995

[23] P. Smilauer, K. Mizushima, and D. D. Vvedensky, Phys. Rev. Lett. 81:5600, 1998 